\title{
The use of liquid-phase method from DMSO solutions for synthesis of CZTS thin film materials
}

\author{
Vladimir V. Rakitin ${ }^{1, *}$, Pavel E. Varushkin ${ }^{1}$, Hao Xin ${ }^{2}$, and Gennady F. Novikov ${ }^{1,3}$ \\ ${ }^{1}$ Institute of Problems of Chemical Physics, RAS, Chernogolovka, Moscow Region 142432, Russia \\ 2 College of Materials Science and Engineering, Nanjing University of Posts and Telecommunications, Nanjing, Jiangsu 210000, \\ P.R. China \\ ${ }^{3}$ Lomonosov Moscow State University, Moscow 119991, Russia
}

Received: 19 March 2019 / Received in final form: 21 June 2019 / Accepted: 18 July 2019

\begin{abstract}
The possibility of synthesis of CZTS thin films on glass substrates from DMSO precursor solutions containing various quantity of copper ions was investigated. The dependence of composition of final CZTS compounds obtained from precursor solutions with different copper ion concentration in DMSO was shown. It was obtained that with the introduction of a low additive of copper ion concentration one can synthesize CZTS thin films with kesterite structure as well as with secondary phases (tin sulfides of $\mathrm{SnS}_{2} \mathrm{Sn}_{2} \mathrm{~S}_{3}$ ). The increase of the concentration of copper ions in DMSO precursor solution promotes the formation of CZTS thin films with kesterite phase and the minimum content of impurities.
\end{abstract}

Keywords: Kesterites / CZTS / thin films / solution / DMSO

\section{Introduction}

The study of kesterite $\mathrm{Cu}_{2} \mathrm{ZnSn}(\mathrm{S}, \mathrm{Se})_{4}$ (CZTSSe) materials has been active in the past 12 years. This is due to the fact that such semiconductors are devoid of a number of disadvantages that other alternative materials have. The CZTSSe semiconductor does not contain rare, expensive or highly toxic elements, and the synthesis technology of this material is favorably distinguished by its inexpensive nature. Kesterite materials have high light absorption coefficient $\alpha \sim 10^{4} \mathrm{~cm}^{-1}$ and optimal band gap $E_{g} \sim 1.0-1.5$. However, the maximum efficiency value of solar cells based on CZTSSe is still 12.6\% [1], which is far from the theoretically possible $32 \%$ [2]. The reason for this may be the presence of secondary phases in the films or molybdenum sulfide at the kesterite/Mo interface and, in general, the relatively less studied formation mechanism of the final films. Therefore, the study of such type of materials shows promising results and is currently an important direction of exploration for researchers.

There are several ways to synthesize kesterite materials. The vacuum and liquid-phase methods are the most often used ones. The most attractive and perspective way is liquid-phase methods because they have a number of advantages in comparison with the vacuum methods, for example: low cost, the possibility of synthesis at room

\footnotetext{
* e-mail: domi-tyan@yandex.ru
}

temperature, and the implementation of deposition on large areas [3-5]. One of the possible and promising methods of liquid-phase synthesis is molecular precursor solution (MPS). So far, a large number of studies of kesterites obtained by liquid-phase methods are published. One can find information about various conditions for synthesis of the kesterite films [6-11]. However, despite the large amount of research in the field of synthesis of CZTS thin-film materials by liquid-phase method, the question of the influence of synthesis conditions on the composition and properties of the final films remains not fully studied. In particular, there is little information in the literature about the effect of the component concentration and, as a consequence, the ratio of elements in the precursor solution on composition of the obtained CZTS films. Therefore, this task is relevant at the moment.

\section{Experimental}

The main technique of synthesis of CZTS films is described in the literature [10]. However, this work did not delve into the influence of the ratio of elements in the precursor solution and only studied the doping effect of lithium in the films on optical and electrophysical properties of the materials. Therefore, this work is planned to investigate the effect of the element concentration of elements in the precursor solution on final composition of kesterite films and their electrophysical and optical properties. 
Table 1. The general parameters of synthesis.

\begin{tabular}{llll}
\hline № synthesis & $m\left(\mathrm{Cu}\left(\mathrm{CH}_{3} \mathrm{COO}\right) \cdot \mathrm{H}_{2} \mathrm{O}\right), \mathrm{mg}$ & $C_{V}\left(\mathrm{Cu}\left(\mathrm{CH}_{3} \mathrm{COO}\right) \cdot \mathrm{H}_{2} \mathrm{O}\right), \mathrm{M}$ & $\mathrm{Cu} /(\mathrm{Zn}+\mathrm{Sn})$ \\
\hline 1 & 798.6 & 0.5642 & 0.5 \\
2 & 912.6 & 0.6448 & 0.57 \\
3 & 1026.7 & 0.7254 & 0.64 \\
4 & 1140.8 & 0.806 & 0.71 \\
5 & 1276.9 & 1.28 & 0.8 \\
6 & 1437 & 1.44 & 0.9 \\
7 & 1596.7 & 1.6 & 1 \\
\hline
\end{tabular}

The synthesis of CZTS was produced from DMSO precursor solution with the addition of copper acetate in various concentrations, as well as zinc and tin chlorides and thiourea. The $\mathrm{Cu} /(\mathrm{Zn}+\mathrm{Sn})$ ratio was varied from 0.5 to 1 (see Tab. 1).

DMSO was chosen as the solvent, as it has a number of advantages from other solvents, such as high polarity (dipole moment $=3.9)$ and high coordination ability for many metal ions.

In the synthesis $1,1140.8 \mathrm{mg}$ of $\mathrm{Cu}\left(\mathrm{CH}_{3} \mathrm{COO}\right)_{2} \cdot \mathrm{H}_{2} \mathrm{O}$ $(99.99 \%)$ and $886.4 \mathrm{mg}$ of $\mathrm{SnCl}_{2} \cdot 2 \mathrm{H}_{2} \mathrm{O}(99.99 \%)$ were dissolved in $5 \mathrm{ml}$ of DMSO (99.99\%, anhydrous) and stirred within a day at room temperature; then $555.0 \mathrm{mg}$ of $\mathrm{ZnCl}_{2}$ was added and stirred until complete dissolution. The solution became brightly green. Further $1560.2 \mathrm{mg}$ of thiourea was put into the solution and a translucent suspension with yellow tint color was obtained. Thereafter the solution was spin-coated on different types of substrates with the rotation speed of $1500 \mathrm{rpm}$ during $60 \mathrm{~s}$. This process was repeated to five times to obtain the total thickness of $2-3 \mu \mathrm{m}$. Finally, the annealing of the synthesized samples in a furnace $\left(550^{\circ} \mathrm{C}\right)$ for $2 \mathrm{~min}$ was conducted. The scheme of the sequential dissolution of components in DMSO media, the process of deposition of precursor solution on the glass substrate and the annealing process are presented in Figure 1.

The synthesis 2, 3, 4, 5, 6 and 7 were similar to the synthesis 1 , but copper concentration and the $\mathrm{Cu}$ / $(\mathrm{Zn}+\mathrm{Sn})$ ratio were changed (see Tab. 1).

The composition of CZTS samples was studied by combination of X-ray diffraction (XRD) with $\mathrm{CuK}_{\alpha}$ line and Raman spectroscopy using a Bruker Senterra microRaman system operating at $532 \mathrm{~nm}$. Determination of band gap energy of semiconductor materials was made according to absorption and reflection spectra obtained by optical spectroscopy using a Shimadzu UV-3101PC spectrometer. The photoconductivity of CZTSe films was determined by the photoelectrochemical method using photoelectrochemical cells with periodic illumination of the sample surface in $\mathrm{Eu}\left(\mathrm{NO}_{3}\right)_{3}$ solution. The illumination of CZTS samples was carried out at intensity of $100 \mathrm{~mW} / \mathrm{cm}^{2}$ and in the potential range of $-1200 \mathrm{mV}$ to $0 \mathrm{mV}$ (vs. $3 \mathrm{M}$ saturated silver chloride electrode (SSCE)).

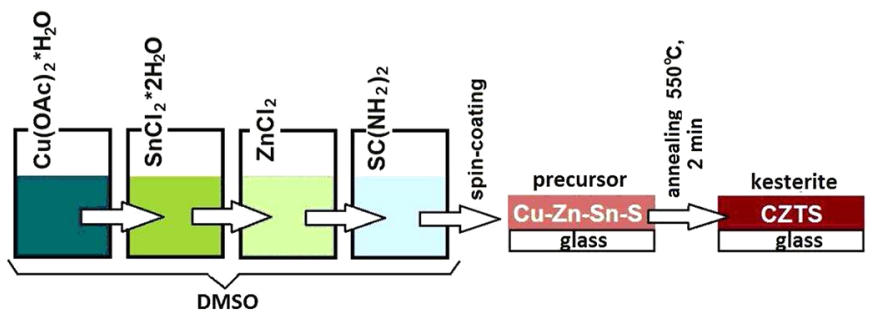

Fig. 1. The scheme of CBD synthesis method.

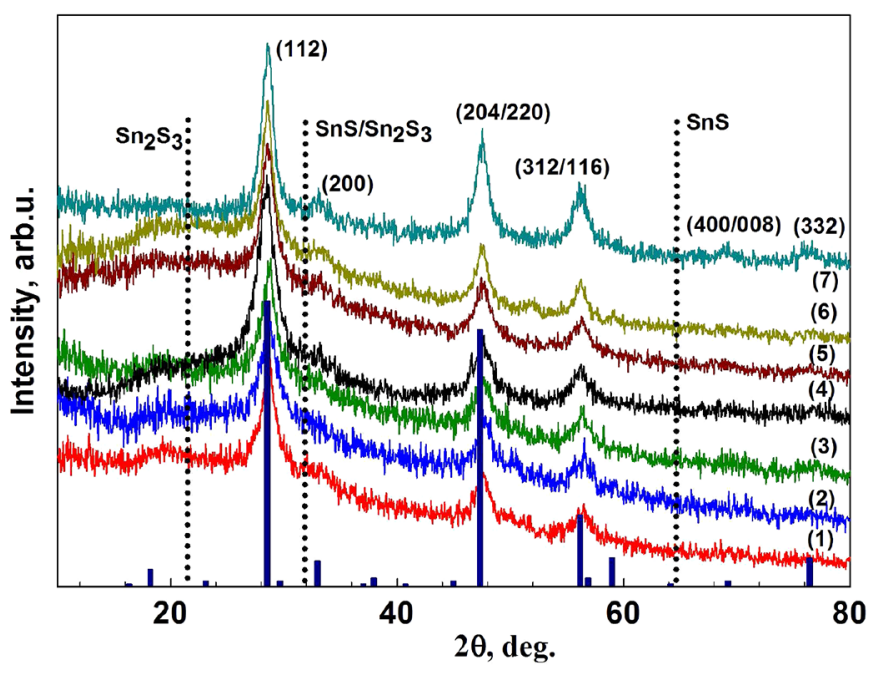

Fig. 2. The XRD data of CZTS samples on glass substrates.

\section{Results and discussion}

\subsection{The structure study of films by XRD method}

Figure 2 shows XRD data for the synthesized samples on glass substrates. The curve number corresponds to the synthesis number in Table. 1.

All curves in Figure 2 have three clear peaks at $2 \theta: 28^{\circ}$ $\{112\}, 47^{\circ}\{220\}$ and $56^{\circ}\{312\}$. These three values correspond to the kesterite phase. The characteristic peaks of standard sample are shown by straight lines. It should be 


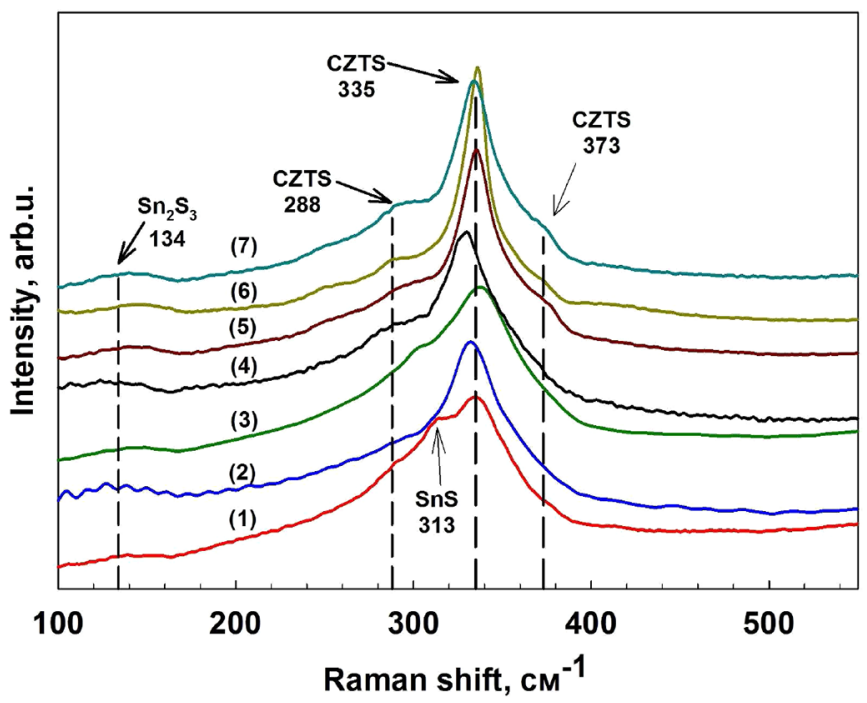

Fig. 3. The Raman data of the CZTS thin films on glass substrates.

noted that the samples synthesized with higher copper concentration (synthesis 7 according to Tab. 1) shows two additional kesterite peaks at $2 \theta: 69^{\circ}\{400 / 008\}$ and $76^{\circ}$ $\{332\}$. This also can be associated with the increase of content of the kesterite phase. The presence of secondary phases ( $\mathrm{SnS}$ and $\mathrm{Sn}_{2} \mathrm{~S}_{3}$ ) in the films was not clearly defined because of their small amounts. The XRD data for the films deposited on glass/Mo and sitall substrates is similar to the above analyzed samples synthesized on glass. With higher copper concentration, samples show additional kesterite peaks at higher angles but peaks related to secondary phases were not clearly detected.

\subsection{The structure study of films by Raman spectroscopy}

To obtain more precise information, Raman data of the samples deposited on all types of substrates were analyzed. The Raman spectra of CZTS samples obtained on glass substrates from the DMSO precursor solutions in the wide range of copper concentrations are presented in Figure 3.

There was no significant difference in the Raman spectra between the substrates of different types (glass, glass /Mo and sitall). As shown in Figure 3 all samples have the main peak at $338 \mathrm{~cm}^{-1}$ which is responsible for the presence of kesterite phase in synthesized films. The second kesterite peak corresponding to value of $289 \mathrm{~cm}^{-1}$ is accurately visible only for the samples obtained at the $\mathrm{Cu}$ / $(\mathrm{Zn}+\mathrm{Sn})$ ratio of $0.71 ; 0.8 ; 0.9$ and 1.0 (synthesis $4,5,6$ and 7 respectively). For the samples obtained from the synthesis No. 3 (for which the $\mathrm{Cu} /(\mathrm{Zn}+\mathrm{Sn}$ ) ratio is 0.64 ), the secondary peak is not intense and is practically absent for the kesterite films obtained from the synthesis No. 1 and No. 2. The kesterite peak at $377 \mathrm{~cm}^{-1}$ appears only for samples obtained since using synthesis $5(\mathrm{Cu} /(\mathrm{Zn}+\mathrm{Sn})$ ratio is $0.8-1$ ). Raman spectra of the films using synthesis 1 also have peak corresponding to tin sulfide phase $\left(314 \mathrm{~cm}^{-1}\right)$ which vanishes in samples with the increase of copper concentration in further synthesis. The main peaks of $\mathrm{SnS}$ and $\mathrm{Sn}_{2} \mathrm{~S}_{3}$ in XRD data coincide with the peaks of kesterite, which confirms presence of this phase in the films.

Based on the results of analysis obtained by combination of XRD and Raman spectroscopy methods, we can conclude that a small content of $\mathrm{Sn}_{2} \mathrm{~S}_{3}$ and $\mathrm{SnS}$ phases was in the obtained films, whereas the presence of copper sulfide phase was not confirmed by Raman spectroscopy. The main Raman peak of $\mathrm{Cu}_{x} \mathrm{~S}$ locates at $494 \mathrm{~cm}^{-1}$ which is not detected in the films.

It is also possible that kuramite phase exists in the films. The main peaks of kuramite phase (Raman shifts at $297 \mathrm{~cm}^{-1}, 337 \mathrm{~cm}^{-1}$ and $352 \mathrm{~cm}^{-1}$ ) are close to kesterite and $\mathrm{SnS}$ peaks both in XRD and Raman spectra. Therefore, negligible content of the impurities in kesterite films cannot be absolutely excluded using the analysis methods mentioned above.

\subsection{The study of optical properties}

Absorption spectra of the samples synthesized on glass substrates were obtained. The dependences in $\left\{(D h v)^{2}\right\}-\{h v\}$ coordinates were calculated according to the obtained data, where $D$ is optical density (see Fig. 4a).

In order to determine the band gap energy of obtained samples, the extrapolation to crossing with abscissa axis was made. The $E_{g}$ band gap of synthesized CZTS films is $1.5 \mathrm{eV}$. There was no direct dependence between changes in the $E_{g}$ values and increase of the copper concentration in precursor solution. The obtained values correspond to the literature data for sulfide kesterite type [6,10-13].

To obtain more precise data about band gap energy of semiconductor materials the reflection spectra of samples synthesized on glass/Mo substrates were also measured. The $\left\{\left(h v^{*} Y(h v)\right)^{2}\right\}-\{h v\}$ dependence was plotted to determine $E_{g}$ value (see Fig. $4 \mathrm{~b}$ ), because rectification in such coordinates is better than in $\left\{(D h v)^{2}\right\}-\{h v\}$ coordinates. Then the extrapolation of straight-line sections of curves to crossing with abscissa axis was made. The $E_{g}$ value varies in the range of $1.5 \mathrm{eV}$, which is comparable to the results obtained from study of absorption spectra.

Such indistinct spectra rectification in $\left\{(D h v)^{2}\right\}-\{h v\}$ and $\left\{\left(h \nu^{*} Y(h v)\right)^{2}\right\}-\{h v\}$ specified coordinates near the red boundary in determination of band gap energy of the final CZTS semiconductor materials may be possible associated with the presence of a small amount of $\mathrm{SnS}$ impurity phase, what is confirmed by Raman spectroscopy. The band gap energy of cadmium sulfide varies between 1.07 and $1.27 \mathrm{eV}$ depending on the thickness (amount of this phase) $[14,15]$ and can contribute to the total band gap energy of CZTS thin films.

\subsection{The determination of conductivity type}

To determine the type of conductivity of the synthesized CZTS thin films, the obtained samples were investigated by photoelectrochemical method using photoelectrochemical cells with periodic illumination of the sample surface. 


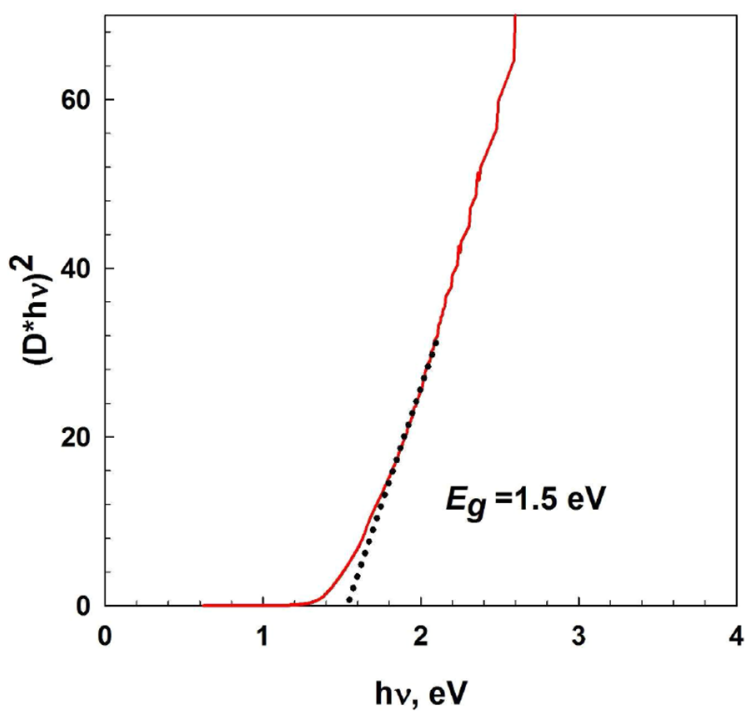

(a)

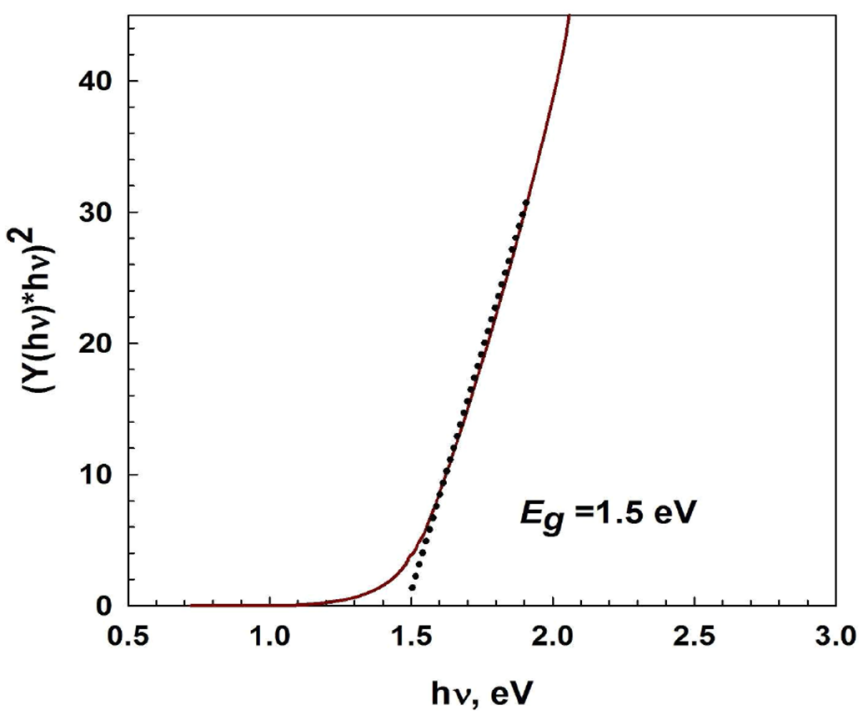

(b)

Fig. 4. The determination of band gap energy according to absorption spectra (a) and reflection spectra (b) of CZTS thin films.

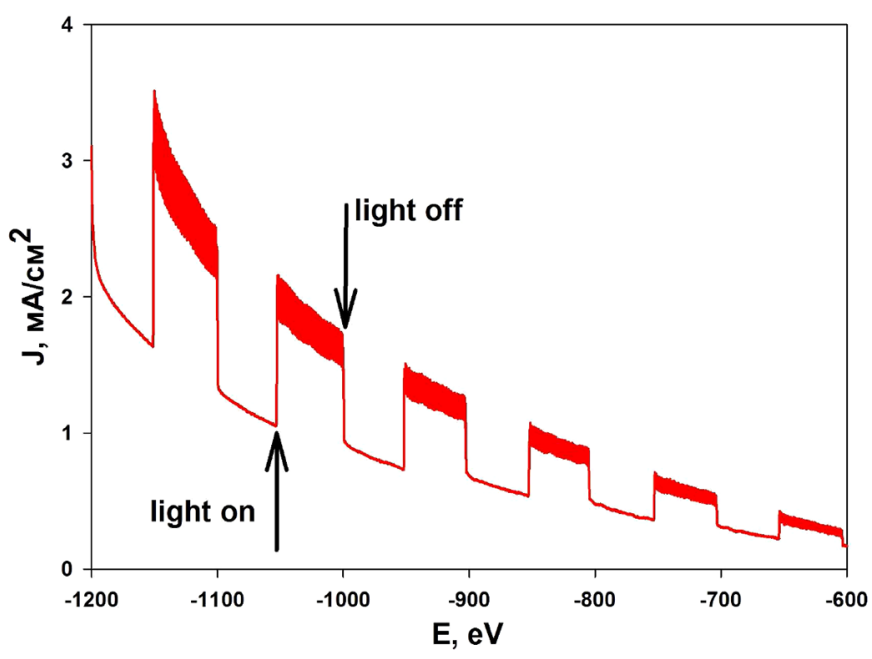

Fig. 5. The current-voltage characteristics of CZTS film (at $\mathrm{Cu} /$ $(\mathrm{Zn}+\mathrm{Sn})=0.5)$ with periodic illumination of surface.

The $J(E)$ dependences were obtained and studied (see Fig. 5). For example, the results of photoelectrochemical measurements are presented on the $J(E)$ dependence for the CZTS sample synthesized at $\mathrm{Cu} /(\mathrm{Zn}+\mathrm{Sn})=0.5$. Characteristic moments of light on and light off are shown by black arrows.

Figure 5 shows that current density increases sharply with the light on, and decreases sharply with the light off. With the direction moving to positive voltage values, the amplitude of current density jumps gradually decreasing in proportion to dark current, as shown in Figure 6, curve 2. In this case, there is a nonohmic current decline (see curve 1 in Fig. 6). The reason is most likely due to the polarization of

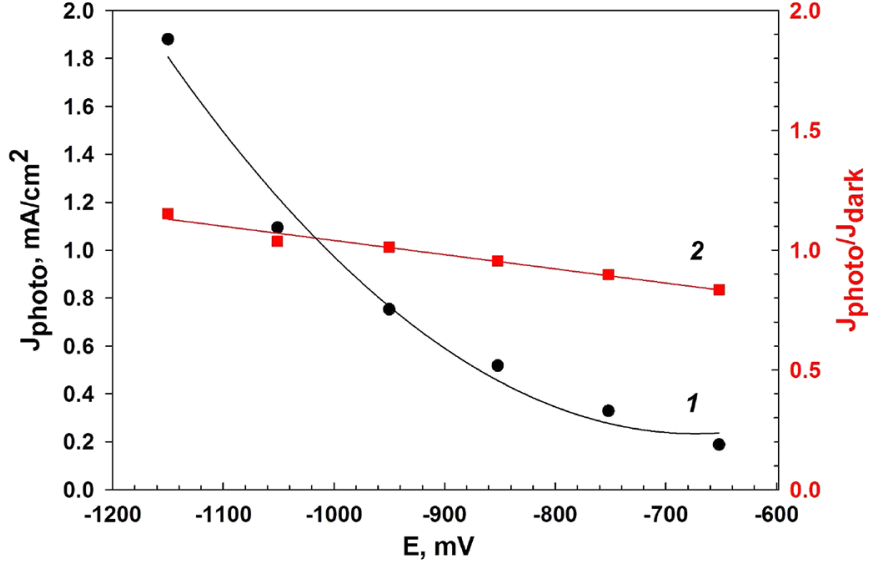

Fig. 6. The dependence of photocurrent density on external voltage for CZTS films with $\mathrm{Cu} /(\mathrm{Zn}+\mathrm{Sn})=0.5$ : (1) photocurrent decay; (2) - normalization at $J_{\text {dark }}$ (solid-line curves are approximations by second order and first order polynomials, respectively).

double-electric layer near the CZTS film surface. Besides, a large number of $\mathrm{Eu}^{3+}$ europium ions collect in near-cathode region at high negative voltage values. In this case, the efficiency of reduction reaction $\mathrm{Eu}^{3+}+\bar{e}=\mathrm{Eu}^{2+}$ are very high and, as a result, the difference between the light and dark curves is large. With potential shift towards positive values, the sorption of $\mathrm{Eu}^{3+}$ europium ions is much less, and the efficiency of reduction reaction is much lower. As a result, the current-voltage dependence shows low jumps, which finally disappear in $0 \mathrm{~V}$ region.

Since the main response to light is observed in the cathode region of potentials, the resulting semiconductor CZTS samples have $p$-type conductivity. 


\section{Conclusions}

Thus, for the system, which proved to be promising for use in thin-film solar cells [10], the influence of synthesis conditions on composition, electrophysical and optical properties of CZTS kesterite films obtained by liquid-phase method from DMSO medium was studied for the first time. The possibility of producing the CZTS thin films with a small content of secondary phases and band gap energy $E_{g} \sim 1.5 \mathrm{eV}$ by liquid-phase method from DMSO solution in the range of copper concentrations $C_{v}=0.56-1.60 \mathrm{M}$ is shown. The combination of XRD and Raman spectroscopy methods revealed that the obtained samples had kesterite type structure with low content of $\mathrm{SnS}$ and $\mathrm{Sn}_{2} \mathrm{~S}_{3}$ impurity phases. It was found that when copper concentration increases in the precursor solution, CZTS films with minimum content of secondary phases are formed. It was determined that CZTS thin films have $p$-type conductivity, and when copper concentration increases in the precursor solution, current passed through the sample increases.

The work was carried out with the financial support of the Ministry of education and science of the Russian Federation: contract No 14.613.21.0065, unique project number RFMEFI61317 × 0065 .

\section{Author contribution statement}

All authors contributed equally to the paper.

\section{References}

1. W. Wang, M.T. Winkler, O. Gunawan, Adv. Energy Mater. 4, 1301465 (2013)

2. V.V. Rakitin, G.F. Novikov, Russ. Chem. Rev. 2, 99 (2017)

3. T.S. Tlemçani, E.B. Benamar, F.Ch. El Moursli, Energy Proc. 84, 127 (2015)

4. S. Shin, Ch. Park, Ch. Kim, Y. Kim, S. Park, J.-H. Lee, Curr. Appl. Phys. 16, 207 (2016)

5. S.K. Aditha, A.D. Kurdekar, L.A.A. Chunduri, S. Patnaik, V. Kamisetti, MethodsX 3, 35 (2016)

6. T. Todorov, O. Gunawan, S.J. Chey, T.G. Monsabert, A. Prabhakar, D.B. Mitzi, Thin Solid Films 519, 7378 (2011)

7. D.B. Mitzi, O. Gunawan, T.K. Todorov, K. Wang, S. Guha, Solar Energy Mater. Solar Cells 95, 1421 (2011)

8. Y.B. Kishore Kumar, V. Sundara Raja, Surf. Interfaces 9, $233(2017)$

9. N.P. Huse1, A.S. Dive1, AIP Conf. Proc. 1832, 080082 (2017)

10. H. Xin, J.K. Katahara, I.L. Braly, H.W. Hillhouse, Phys. Chem. Chem. Phys. 17, 23859 (2015)

11. H. Xin, J.K. Katahara, I.L. Braly, H.W. Hillhouse, Adv. Energy Mater. 4, 1301823 (2014)

12. S.S. Fouad, I.M. El Radaf, P. Sharma, M.S. El-Bana, J. Alloys Compd. 757, 124 (2018)

13. J. Li, Y. Zhang, Y. Wang, C. Xue, J. Liang, G. Jiang, W. Liu, C. Zhu, Sol. Energy 129, 1 (2016)

14. S.A. Bashkirov, V.F. Gremenok, V.A. Ivanov, Semiconductors 45, 749 (2011)

15. S.A. Bashkirov, P.P. Gladyshev, V.F. Gremenok, V.A. Ivanov, Phys. Solid State 56, 1310 (2014)

Cite this article as: Vladimir V. Rakitin, Pavel E. Varushkin, Hao Xin, Gennady F. Novikov, The use of liquid-phase method from DMSO solutions for synthesis of CZTS thin film materials, EPJ Photovoltaics 10, 6 (2019) 